Review Paper

\title{
Generation and Applications of Plasma (An Academic Review)
}

Faizan Mehmood ${ }^{1}$, Tariq Kamal ${ }^{2}$ and Umair Ashraf ${ }^{3}$

1 Department of Electrical Engineering, University of Engineering \& Technology, 47080, Taxila, Pakistan; faizanmehmood.ee@outlook.com/㐌faizan024@gmail.com

2 Research Group in Electrical Technologies for Sustainable and Renewable Energy (PAIDI-TEP-023), Department of Electrical Engineering, University of Cadiz, Higher Polytechnic School of Algeciras, 11202

Algeciras (Cadiz), Spain and Department of Electrical and Electronics Engineering, Sakarya University, Turkey; tariq.kamal.pk@ieee.org

3 Department of Earth and Environmental Science, Bahria University, Pakistan; umair.ashrf@gmail.com

* Correspondence: faizanmehmood.ee@outlook.com; Tel.: +92-335-561-1708

\begin{abstract}
Plasma being the fourth and most abundant form of matter extensively exists in the universe in the inter-galactic regions. It provides an electrically neutral medium of unbound negative and positive charged particles, which has been produced by subjecting air and various other gaseous mixtures to strengthen the electromagnetic field and by heating compressed air or inert gasses for creating negative and positive charged particles known as ions. Nowadays, many researchers are paying attention to the formation of artificial Plasma and its potential benefits for mankind. The literature is sparsely populated with the applications of Plasma. This paper presents specific methods of generation and applications of Plasma, which benefits humankind in various fields, such as in electrical, mechanical, chemical and medical fields. These applications include hydrogen production from alcohol, copper bonding, semiconductor processing, surface treatment, Plasma polymerization, coating, Plasma display panels, antenna beam forming, nanotechnology, Plasma Torch, Plasma pencils, lowcurrent non-thermal Plasmatron, treatment of prostate cancer, Plasma source ion implantation, cutting by Plasma, Plasma etching, pollution control, neutralization of liquid radioactive waste, etc. Resultantly, worth of Plasma technology in the medical industry is increasing exponentially that is closing the gap between its benefits and cost of equipment used for generating and controlling it.
\end{abstract}

Keywords: Plasma Generation Techniques, Applications of Plasma; Fusion technology; Plasma ion implantation and Deposition.

\section{Introduction}

Plasma has been observed by mankind in nature since a long time ago. It has recognized as the $4^{\text {th }}$ state of matter. Most of the universe comprises the Plasma rather than solid, liquid or gas. Astrophysicists found that galaxies are mostly composed of this form of matter. The Plasma term was initially recognized by a medical scientist, Purkinje (1787-1869) that referred it as the clear liquid, which is left after various corpuscle components, and protoplasm are removed from the blood, but this was not the Plasma, which is nowadays recognized as the $4^{\text {th }}$ state of matter. This was first ever discovered by William Crooks in 1879, and this state of matter was officially named as 'Plasma' by Irving Langmuir in 1928.

The theory of Plasma Sheath was developed by Langmuir, which is the boundary layer formed between solid particles and ionized Plasma. He also discovered the periodic variations of electron density in certain regions of Plasma discharge tube, which is known as Langmuir Waves. This was how Plasma physics came into being. 
Plasma mainly constitutes of negative ions, and heavy charged particles. Therefore, Plasma can be defined as partially ionized gas usually produced by an electrical discharge at near surrounding temperature. Due to extensively increasing potential and benefits of Plasma in recent years, the application of Plasma is growing very rapidly.

Scientists ever tried to model this form of matter and some equations defining this state. These equations apply to many proposed controlled thermo-nuclear reactors. Many issues regarding this state of matter were raised in past like the mere fact of localization of the Plasma anisotropic heating. To resolve such concerns, various models were already developed. These involve models, which gives information about the frequency dependence of the resistivity, and the associated processes of absorption and emission of radiation and effect of frequencies [1-3].

Nuclear Physics usually require slow neutrons for many of its operations like fission and fusion reactions. Plasma also finds its role in nuclear physics. Later studies revealed that Plasma also exists in several other forms like Non-equilibrium Plasma, which is initiated as the appropriate medium for surface properties modification of the material surface occurs. To build an effective treatment with energy and time-saving feature, various Plasma parameters are used that depends on the type of materials and their properties. Such treatment properties were considered as to be drawn of organic chemistry, during the late $20^{\text {th }}$ century. The implication and its efficient use for synthetic fabrication make it much famous in various industrial products. In the field of artificial products, this also provides the platform for advanced research in the field of organic materials integration and control. Moderate and highly ionized Plasma can also be able to provide temperature resistive effects for inorganic materials like ceramic, alloys and glass.

Plasma used in medical application occurs during the biological and chemical distillation, Plasma therapy, sterilization and the blood coagulation process. It also provides the ease of producing low-temperature Plasma in the open air.

Use of Plasma technology for the efficient development of Inter-punitive sciences can efficiently provide the equipment's designing for effective diagnoses and monitoring of diseases level and tools used during the medical process. According to reviews of pharmaceutical scientists about the use of Plasma for diagnoses of diseases, they provide several positive effects for Plasma use. Like the scientist, Stoffels in [4], states it as the invention of Plasma needle, which provides a practical use during the treatment of living tissues. Moreover, many other applications of Plasma in the medical field will be discussed later.

In the future, a key problem to be faced will be a lack of energy resource for power generation. Scientists frequently consider the use of alternative energy resources, in which the most important practical method is the use of Plasma under the field of power production. It also identifies the implication of newly exposed state of matter and discovers the dynamic behaviors to integrate it into the electrical system, one good example is a portable Plasma torch. Electrical scientists also identify the significance of this newly discovered state of matter and find ways to integrate it into the electrical systems, like in the portable Plasma Torch [5].

\section{Categorization of Plasma}

The process for plasma generation requires the application of energy on gas, which causes ionization of gas [6]. According to the temperature of electrons, Plasma is classified into two groups, non-thermal (cold) and thermal (hot) plasma based on their degree of ionization, atmospheric pressure and the temperature conditions [7-13].

i. Hot (thermal) Plasma is composed of negative ions and heavy charged particles, which are in thermal equilibrium with each other, i.e., both, are at the same temperature. Thermal Plasma is thermodynamic and can abundantly found in the universe [14]. It can be produced by electrothermal and electromagnetic launchers, (Lehrstuhl fur Raumfahrttechnik) [15], investigated the micrometeoroid and space debris impacts for surface treatment to provide Plasma of hot, dense, short wavelength and 
high velocity by the accelerated small glass beads that can directly affect the material surface for coating purpose. The use of Plasma antenna for beamforming has the interaction of Plasma elements which includes various scattering from Argon Plasma cylinders boundary and electromagnetic wave transmission on Argon Plasma, due to the incident electromagnetic wave for beam formation of the antenna. Such Plasma antenna provides highly efficient beam radiation, beam forming and beam scanning [16]. Ion implantation based on Plasma is an advanced technique for depositing thin films and surface modification for many fabrication processes in industry.

ii. Non-thermal Plasmas (Cold Plasma) have electronics which are at much higher temperature than the heavy charged particles. Cold Plasma manifests a behavior outside the thermodynamical equilibrium [17]. The conventional ion sources include Plasma generators for ion extraction and acceleration. The most commonly used techniques in Plasma generators to create discharge are electron cyclotron resonance and microwave frequency ionization source [18].In recent years, the technology to produce Plasma at high temperature without gaseous collision has been of interest due to its demand in space physics, inertial fusion and magnetic fusion [19]. The frequency and power of radiation emitted by artificial Plasma can be modified by using vacuum microwave oscillators as ionization sources [20]. Plasma gun technique for high energy density Plasma contains electrodes connected with high current sources like MARX generators or magnetic flux compression generators in order to ionize the gas injected and foils to accelerate the ions by Lorentz force, which then results in expulsion and flow of resultant Plasma [21].

Plasma technology is growing rapidly for industrial as well as commercial applications. Plasma technology has a strong application for use in metallurgy process, nanotechnology tools [22] for forming the antenna beams. Its application in Plasma lenses ensures the biocompatibility with the human tissues for treatments of living cell tissues [23]. The surface treatment application or the implant material treatment that is targeting the blood coagulation or wound healing aid for avoiding the electrocution, the preferred one would be the floating potential electrode [17] [12]. Its use in reactors with corona discharges and Plasma display panels is much efficient than the conventional flat screen televisions due to the development of better performance, highly efficient, and low-cost advanced electronic circuitry driver topologies. It depends on the resonant energy source, resonant network connection type, and panel voltage levels [24]. The literature published on the use of Plasma in the processing of semiconductor is currently far better than the published on its use for fusion research [19]. The purpose of this paper is to provide an idea of the development, advancement and applications of Plasma, which will be discussed in following sections.

\section{Plasma Generation Techniques}

i. DC Glow Discharge: This belongs to non-thermal plasma, in which Direct current (DC) electric source is connecting between cathode and anode plate and the application of plasma gas is performed among the plates for plasma generation.

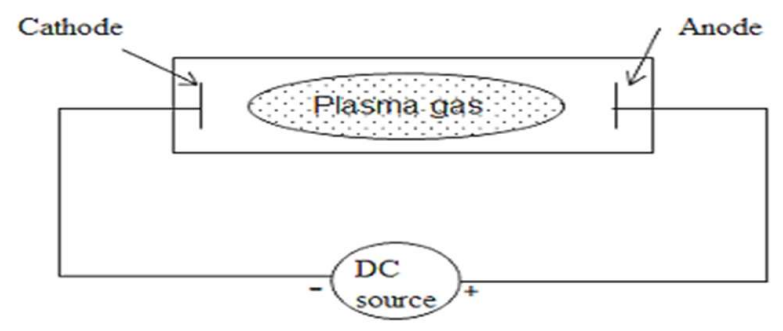

Figure.1 System for DC glow discharge 
The application of DC electrical field across the cathode and anode plate causes the acceleration of electrons in the front end of the cathode, which increases the inelastic collision between atoms and electrons and leading towards the ionization and excitation. Ions and new electrons created by the ionization collision are strongly accelerated by the electric field toward the cathode that discharges the new electrons by ioninduced through the secondary electron emission. The increase ionization collisions increase the concentration of new electrons and ions at the cathode build the discharge glow of self-sustaining plasma. The emitted electrons from the electrodes usually unable to sustain the discharge when there will be no potential difference between the electrodes, while the injection of constant potential difference increases the DC discharge because of the large flowing of current. This can be extensively utilized for material processing, as a light source, etching, Ion-deposition and for physical mechanism of surface modification.

ii. Radio Frequency Discharge: It produces the plasma either by inductively or capacitively coupling energy at frequency range lies under the radio spectrum $(1 \mathrm{KHz}$ to $103 \mathrm{MHz})$ and has $\mathrm{AC}$ power supply. Based on the coupling mechanism, there exist two kinds of RF plasma discharge, i.e., the Capacitively Coupled Discharge (CCD) and Inductively Coupled Discharge (ICD). Within capacitively coupled discharge system, the AC voltage source is provided to power electrodes through a capacitor, while the other electrode is solidly grounded. The capacitor rapidly charged at the positive half of the voltage source, which causes the voltage drop over the plasma. The charging up of the capacitor by the ion current and dropping of plasma voltage likely occurs in the negative half cycle but the voltage is much prevalent of the lower ion mobility. It preferably utilizes as a lower temperature processing medium of plasma for material processing in aerospace and microelectronics fields.

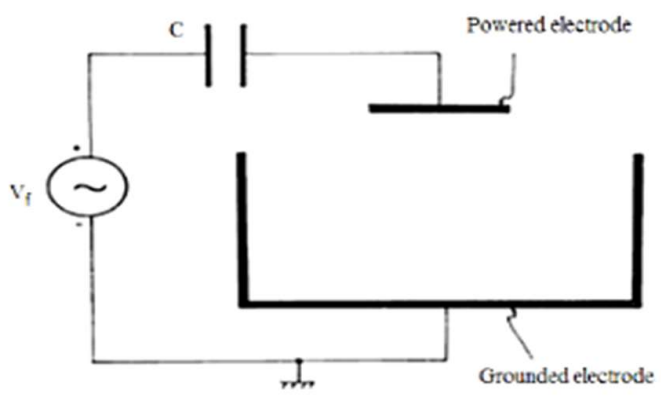

Figure.2Capacitively coupled discharge system

The inductive coupled discharge contains the configuration of a cylindrical helical coil, in which the electromagnetic induction provides the corresponding electric current. The passing of RF current through the coil develop the time-varying magnetic flux that largely induces the RF sinusoidal electrical field that sustains the plasma discharge and accelerate the free electrons.

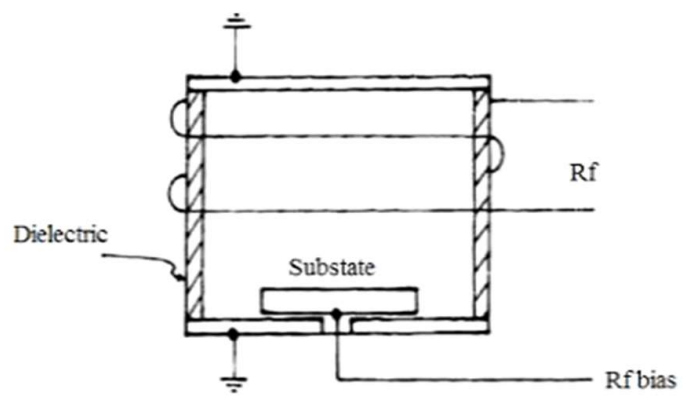

Figure.3 Inductively coupled discharge system

Transformer coupling usually aid for inducing the electromagnetic field between the induction coil and plasma at a frequency range of 1 to $100 \mathrm{MHz}$ It is much efficient for generating the oscillation and electron acceleration 
in the curved orbits that cause a significant decrease of electron losses with the walls. It is preferable to use in deposition and etching for processing of semiconductor wafers, for modifying the surface surfaces of diamond films and fabrication purposes.

\begin{tabular}{|c|c|c|c|}
\hline Source of Plasma & $\begin{array}{c}\text { Background } \\
\text { pressure/Torr }\end{array}$ & $\begin{array}{c}\text { Electron number } \\
\text { density/m }\end{array}$ & Nominal Power / W \\
\hline RF glow discharge & $0.05^{-1}$ & $10^{17}$ & $200-500$ \\
\hline DC glow discharge & $0.05^{-5}$ & $10^{16}$ & $100-300$ \\
\hline Inductively Coupled & $10^{-3}-0.1$ & $10^{18}$ & $500-2000$ \\
\hline $\begin{array}{c}\text { Electron cyclotron } \\
\text { resonance }\end{array}$ & $10^{-4}-0.01$ & $10^{18}$ & $300-1000$ \\
\hline DC welding arc & 750 & $10^{19}-10^{23}$ & $500-2000$ \\
\hline Helicon & $0.01-0.1$ & $10^{18}-10^{19}$ & $500-2000$ \\
\hline DC plasma jet & $750-75000$ & $10^{20}-10^{23}$ & $1000-20000$ \\
\hline
\end{tabular}

Table.1 Plasma generation techniques and their properties

\section{iii. Hydrogen Production from alcohols}

Non-thermal Plasma has more applications in the industry than thermal Plasma. Methanol has high $\mathrm{H}$ to $\mathrm{C}$ ratio. Methanol can be made from methane, which is abundant. Conversion of methanol to hydrogen can be made done by Dielectric Barrier Discharge (DBD) Plasma, corona discharge Plasma, surface-wave discharge Plasma, Microwave Plasma, glow discharge Plasma, pulse charge Plasma, etc. [6]. DBD is also known as silent Plasma. It is generating by using the two electrodes, which have a barrier between them made up of dielectric of few millimeters in thickness. Three different variants of the setup used for making DBD Plasma are shown in Fig. 4.

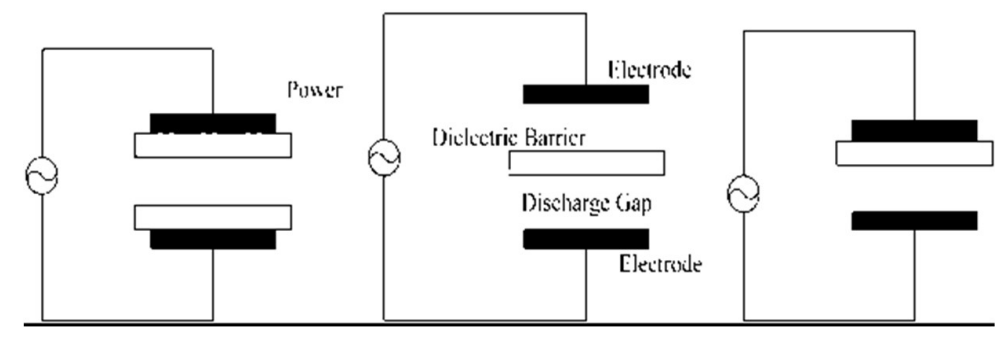

Figure.4 Three basic configurations

The useful product from the decomposition of methanol is hydrogen and byproducts are $\mathrm{CO}$ and $\mathrm{CO}_{2}$. The highest yield of hydrogen achievable by this process to date is about $28 \%$ [25].

$$
\begin{aligned}
& \mathrm{CH}_{3} \mathrm{OH} \rightarrow 2 \mathrm{H}_{2}+\mathrm{CO} \\
& \mathrm{CH}_{3} \mathrm{OH}+\mathrm{H}_{2} \mathrm{O} \rightarrow 3 \mathrm{H}_{2}+\mathrm{CO}_{2}
\end{aligned}
$$

\section{Applications of PLASMA}

Some of the Plasma applications for both the industries as well as individual users are as follows:

\section{i. Pollution Treatment}

Diesel engines are used in a different application like in transportation, power generation, farming, construction and in other industrial settings. They produce a significant amount of pollution, especially NOx [26]. NOX storage reduction, Selective NOX recirculation and Non-thermal Plasma have been considered increasingly in recent times, with a view to developing techniques to reduce $\mathrm{NO}_{\mathrm{X}}$ emissions in diesel engine [26]. Non-thermal Plasma technology has been introduced as a promising method of $\mathrm{NO}_{\mathrm{X}}$ removal which is done by reaction with free electrons, ions, radicals, molecules in Plasma [27]. NOX removal can be summarized as the following: 


$$
\begin{aligned}
& \mathrm{N}_{2}+e \rightarrow \mathrm{N}+\mathrm{N}+e \\
& \mathrm{NO}+\mathrm{N} \rightarrow \mathrm{N}_{2}+\mathrm{O} \\
& \mathrm{O}_{2}+\mathrm{O} \rightarrow \mathrm{O}_{3} \\
& \mathrm{~N}_{2}+e \rightarrow e+\mathrm{N}_{2}(A) \\
& \mathrm{N}_{2}(A)+\mathrm{NO} \rightarrow \mathrm{N}_{2}+\mathrm{N}+e \\
& \mathrm{~N}_{2}(\mathrm{~A})+\mathrm{N}_{2} \mathrm{O} \rightarrow \mathrm{N2}_{2}+\mathrm{O} \\
& \mathrm{N} 2_{2}+\mathrm{N} \rightarrow \mathrm{N}_{2}+\mathrm{O}_{2}
\end{aligned}
$$

Where $\mathrm{N}_{2}$ (A) represents $\mathrm{N}_{2}$ meta-stable state.

\section{ii. Liquid radioactive waste utilization}

Plasma technology can be used for the utilization and neutralization of liquid radioactive waste [28]. The most difficult phase of the nuclear fuel cycle is safe disposal or recycling. This requires separation and extraction of different components to regenerate the irradiated fuel, and neutralization of waste before disposal. The irradiated fuel is around $97 \%$ and waste is around $3 \%$, which consists of $U_{235}$ and radioactive isotopes of plutonium [29$30]$.

\section{iii. $\quad$ Semiconductor Processing}

Today about a hundred chips are made from a silicon wafer of 4-8-inch diameter. The element forming these chips should be around 0.25 micrometer [19]. This resolution is only possible with Plasma. The Plasma helps in the process of etching in following ways

a) Atomic species such as fluorine or chlorine are produced using Plasma which in turn perform the etching

b) A substrate is produced by using Plasma so that the etchant species act in an effective manner.

c) Plasma helps to keep the process of etching in a straight line due to its highly directional properties

\section{iv. Ion Implantation}

Ion implantation is a process in which the acceleration of ions towards the targets occurs at high energies to enter them below the surface of the target that usually depends on the acceleration energies and the applications for which it is used [31]. Ion implanters are used for the manufacturing of modern integrated circuit (IC) by modifying silicon or doping several other semiconductors. It includes the production of an ion beam and it is steering into the substrate in order to make the ions to come to rest under the surface. The beamline at energy causes the ions to move at which they were taken out from the source material or energy at which they are decelerated or accelerated by radio frequency or dc electric fields.

\section{v. Living Tissues Treatment}

The Cold Plasma treatment initially dedicated to environmental protection has been completed in the last years with bio-medical and bio-decontamination treatments. The living tissue treatment imposes characteristics for the devices used to produce cold Plasma such as;

a) To avoid the electrocution.

b) Reduction of invasive actions on living cells.

c) Assuring the selectivity to affect only the afflicted cells.

d) Avoiding the thermal effect.

e) Limitation of treatment time to avoid the induction of toxic effects to treat cells [17].

\section{vi. High Energy Density Pinch Plasma}

The synthesis of different characteristics Nano-scale materials have many optimizations and improvement processes and routes, including vapor reduction and milling, chemical and nature's organic routes. Lowtemperature (cold) Plasmas have temperature and density very smaller than high-density (hot) pinch and fusion 
plasma. Very high temperatures present in pinch and fusion plasma can produce the fully ionized material, while the low-temperature Plasmas is produced by DC or AC energization of working gas, radio-frequency or microwave electromagnetic fields. Such discharge level has a low level of ionization and most of the ions still exist in the neutral state, having the energy of few tens of electron volts that use kinetic temperatures of an electron to produce Nano-materials [32-34]. Low-temperature plasmas are many times preferable for use in nanotechnology applications. These can be further classified as non-equilibrium (non-thermal) and equilibrium (thermal) Plasmas. The Non-equilibrium plasma follows the relation of $T_{e}>T_{i}=T_{g}$. Where $T_{g}, T_{e}, T_{i}$ and are corresponding temperatures of background gas, ions and electrons. These values are dependent on kinetic energies.

Generation Process of non-equilibrium thermal Plasmas are followed:

a) Low operating pressure technique which results in less frequent collisions between neutral, ion and electrons and hence they cannot achieve thermal equilibrium.

b) The high-pressure techniques used along with pulse discharge. The collisions are high but for short time, so the thermal equilibrium cannot be reached due to pulse discharge interruption [35].

c) The high-pressure micro-plasma is high-pressure plasma whose dimensions reduced to micron size, which causes enormously increase in electrons kinetic energy due to the high electric field.

The equilibrium (thermal) Plasmas are in thermal equilibrium where temperature equilibration made by an electron, ions and neutral repeated collision in high-pressure plasma discharges results in the substantial degree of ionization due to $\mathrm{T}_{\mathrm{e}}=\mathrm{T}_{\mathrm{i}}=\mathrm{T}_{\mathrm{g}}$. Both equilibrium and non-equilibrium techniques have been widely used in Plasma Nano-technology. The thermal, chemical and electrical properties of low-temperature Plasmas provide an efficient and versatile tool for nanotechnology [36].

\section{vii. Plasma Pencil}

Plasma pencil, which is also known as the Plasma plume is considered as the most important application for low pressure or non-equilibrium plasma. In the medical field, low-temperature plasma provides the no arcing risks and generates a low concentration of ozone because of plasma formation [37].

It is frequently used for healing wounds, kill the bacteria inside the mouth and modify the surface of heat sensitive materials. Plasma plume usually creates the Cold Plasma that destroys the bacteria without damaging the skin tissues.

Plasma plume contains the two electrodes of copper rings that are attached to the glass disk surface having a hole in the center of about $3 \mathrm{~mm}$. Power supplied is high voltage pulse with very high frequency (in $\mathrm{kHz}$ ) and is applied to electrodes when gas is inserted. The device usually works on 15-watt DC power supply. The gas used in this application is mainly Helium gas with small traces of Oxygen gas. The Plasma plume production by this method is around $5 \mathrm{~cm}$ in length, which can increase up to $12 \mathrm{~cm}$ in length. Key factors that can affect the Plasma plume length largely depend on the flow rate of Helium gas through the electrodes and the magnitude of voltage pulses.

Substantial disadvantages of other Plasma jet producing devices are that Plasma pencil causes several degrees rises of temperature from the room temperature, which can make unsafe for the skin. In addition, in Plasma jet devices, the plasma length can also be reduced to about few millimeters [38].

\section{viii. Low Current Non-Thermal Plasmatron}

Hydrogen gas has many important industrial uses, such as used as an efficient power source in aerospace for hydrogen gas fuel rockets and the automobile industry, etc. It is also a useful source of energy in a fuel cell or thermonuclear fuels. It has found its uses in fertilizers, petroleum refining, food and fat processing also [39]. One of the most effective way of producing Hydrogen gas is by using Low Current Plasmatron which is also an energy efficient way. Conventional sources used to produce Hydrogen require very large plants; high cost 
and catalysts depreciate very quickly [40]. The new Plasmatron has $70 \%$ conversion efficiency and produces $3 \%$ less heat.

\section{ix. Use of Plasma in Treatment of Prostate Cancer}

In the early days, curing cancer was a matter of early detection and timely treatment of the tumors. The side effects of techniques used to treat the cancerous cells were quite a few including the damaging of body cells without the assurance about the complete elimination of cancer [41].

Low-temperature Atmospheric Plasma has revolutionized the field of medicine and is being successfully used to cure cancer. It has low side effects than the conventional techniques and the chances of cancer returning to the body are minimal. The reason is that the low-temperature plasma breaks down the DNA double strands within the nucleus of Prostate cancer [42].

The device uses $6 \mathrm{kV}$ supply at $30 \mathrm{kHz}$ frequency. Helium gas is used with $0.3 \%$ of Oxygen gas mixed with it. The distance of the plume is $10 \mathrm{~mm}$ from body to nozzle and the time of operation is usually 5 to 10 minutes. Body temperature does not go above $36.5^{\circ} \mathrm{C}$ during that period [43].

\section{x. Cutting by Plasma}

Plasma can be used to cut the materials also. We have been using it for many years, but it is cost-effective and depend on the range of the material. In the start, the cutting system had some limitations just, because, of the thickness of material but now, the quality of cutting system has been improved [44]. There are two machines used to cut materials as CombiCut and RUR machines.

We can see the significant improvements in the cutting system of Plasma. Holes for the bolt can easily be driven and the edges are made excellently with this system even with the thicker material [45]. High definition Plasma cutting is best for stainless steel cutting, mild steel cutting and aluminum cutting.

\section{xi. Plasma Etching}

Sputtering or ion milling is used for etching, the item that is being etched is kept on one side of the electrode of the system. Ions are accelerated by DC radio frequency or microwave electric field. To increase Plasma density, a magnetic field is used. We can also use inert gasses or reactive gasses for this purpose.

Plasma that is generated from RF has a frequency ranging from $\mathrm{kHz}$ to $\mathrm{GHz}$. Plasma is produced from the region that is empty of the substrate and then defused so that it doesn't cause any harm to the substrate by high energy electron [46].

Plasma etching is used in silicon dioxide for making memory devices. Plasma gives high-ranking results for chemical etches (especially wet) [47]. Numbers of gasses are used for Plasma etching some of them are SF6, CF4, Halogen mixtures (CF4/O2). The Plasma has the ability to break these gasses into reactive ions, neutrals or free radicals then they interact with the substrate as they both play their role in etching, especially neutrals. Reactive Ion Etching (RIE) tools are given in Figure. 5.

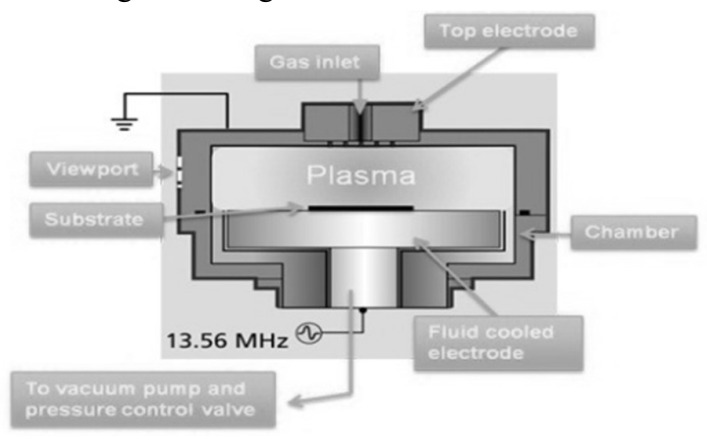

Figure. 5 Reactive ion etching tools

\section{xii. Surface Treatment}


It is named as Glow discharge cleaning and it is like sputtering, but it removes the impurities from the surface of the material so this process is used to clean the things i.e., medical instruments and vacuum surfaces [15]. PIII we can also say Plasma Immersion Ion Implantation, this is used to harden the surfaces, so we can improve the resistance to wear fatigue and corrosion as a ferrous material that is hardened by Nitriding. PIII uses Plasma as the source of ions that is being embedded. The substrate is dipped then with proper biasing ions is rushed to the substrate with proper energy [48].

\section{xiii. Plasma Antenna of Beam Forming}

This Plasma based beam formation and radiation technique used widely in communication [49-50], radio detection, target directing [51]. In the communication field, the engineers use Plasma element as an effective radiator (Omni-directional radiation source) for electromagnetic energy release. In radio detection and target directing technique, we consider Plasma element as a planar shape reflector to reflect the electromagnetic wave in a specific direction [52]. Plasma element can be easily energized by high-frequency AC discharging in vacuum tubes other than a heavy big size cylinder. The cylindrical geometry reflector can also be used other than to planar shape to scatter wave energy and then concentrate it in the predicted direction.

To prevent the leakage of electromagnetic energy by the penetration of Plasma shield we consider the radiation source frequency well below the Eigen frequency for excellent performances in term of beamforming, beam scanning and radiation [53]. This Plasma antenna technique has great influence on Plasma density, Plasma collision, radii and separations of the Plasma elements.

\section{xiv. Atmospheric Pressure Plasma Jet}

The conventional low-pressure plasma process provides a directional etching and deposition of thin films at a rate of $10 \mathrm{~m} / \mathrm{min}$ with temperature as below as $150^{\circ} \mathrm{C}$, so it prevents the damage of thermally sensitive substrates. A uniform glow discharge generation provides the material processing at the same rate over large substrate areas. Vacuum systems are very expensive because it require a special arrangement for its operation and its maintenance is very costly, but this efficient method has high applications like load locks and robotic assemblies used for shuttle materials, also the size of the object used for operation is limited by the size of the vacuum chamber. The atmospheric pressure plasmas techniques overcome the disadvantages of vacuum operation but it is very difficult to support a glow discharge because higher voltages are necessary for the breakdown of gas at 760 torr, which often causes arcing between the electrodes [54]. Plasma torches application produce arcing which can be easily sought, however, to prevent arcing and to low the temperature of the gas, pointed electrodes in corona discharges [55], or insulation insertion in dielectric barrier discharges are used [56]. However, still, the problems of uniformity throughout the volume occur in these techniques.

A Plasma jet based new technique is developed most recently that uses helium flow and unique electrode design to prevent arcing [57], which can etch and deposit materials at low temperatures for a wide range of applications.

\section{xv. Plasma gun Techniques for Fusion at MegaGauss Energy Densities}

A simple controlled fusion Plasma gun technique is used to obtain high-energy ions but has some problems like adequate quality for Fusion Plasma (electrode ablation), separation of reacting Plasma from the permanent portion of the system and repetitive operation for fusion power reactors [58].

The more advanced technique includes the coaxial guns in the form of the Plasma Flow Switch (PFS) that operated through the wire arrays discharge and foils in vacuum to build up the magnetic energy in very lowdensity plasma and then release this magnetized plasma at very high speeds. The PFS technique generates fusion temperature Plasma from an ultrahigh speed flow [59]. This high-speed electromagnetic force has applications in Plasma physics community in controlled fusion research. To provide converging, collimated jets to compress 
the Plasma generators and chamber walls a spherical array based quasi-steady plasma guns technique are used, but it is critical for the development of the pulsed system.

\section{xvi. Plasma Ion Implantation and Deposition}

In Plasma-Based Ion Implantation (PBII) and Plasma Based Ion Implantation and Deposition (PBIID) techniques, the target uses as a part of the beam forming system, in which ions beams are generated and used. The deposition is an integral part of the treatment process. These Plasma immersion ion implantation and deposition techniques suitable for an innovator non-line of sight process by incorporating a three-dimensional shaped target (substrate) in the ion acceleration scheme, the treated object immersed in Plasma and by biasing it became the part of ion source [60]. The biased target surface formed around the ion acceleration that occurs in a dynamic, self-adjusting sheath.

These technologies have tribological applications by decreasing wear and corrosion through the development of hard, tough, low-friction, smooth and chemically inert phases and coatings, which majorly used as frictionless engine components and have applications in microelectronics [61]. Fundamental physical and economical limitations occur in conventional technique due to increasing of size, a number of substrates and pulsed high voltage reached at a level of $100 \mathrm{kV}$, which yield large secondary electrons causing disadvantages. The great technical challenges and high-cost issue leads due to the pulse modulator to handle the total current, which is dominated by secondary electrons rather than ions. Generally, the shielding against X-rays is required due to the impact of high-energy electrons with the chamber wall or various other components. It causes an increase in the process cost [62]. For this; the modern techniques of PBII and PBIID require a few kilovolts voltage level or even less where pulse modulation is affordable. Therefore, it can be effectively used in large area processing emerging fields like biomaterials, deposition of thin films with controlled stress, and synthesis of nanostructured thin films [63].

\section{xvii. Electrothermal and electromagnetic Plasma for surface treatment}

These launchers produce Plasma of hot, dense, short wavelength and high velocity by the accelerated small glass beads that can directly affect material surface [64], so we use it directly for coating purpose in which specific properties of some material impose on other material to achieve better properties of them and neglect bad characteristics. Such interface Plasma pulses on metallic surfaces produce an extremely rigid metallic surface that has very high tendency to resist the corrosion [65-66].

The experiments performed on LRT launcher shows that the resistance to friction corrosion considerably increases during Plasma pulse treatment process which causes no oxides on the treated surface layer of the material, for that electromagnetic and electrothermal launchers can be used [67-69].

\section{Conclusions}

Plasma technology is the most challenging field of research, which has a wide range of applications but still has some unsolved issues. Many issues are expected to be solved in the near future as the research is going on. Understanding Plasma, its applications and problems require knowledge of many areas like particle and radiation physics, electronics, electromagnetic wave theory, thermodynamics, quantum mechanics, physical chemistry and many others. The future of Plasma technology is anticipated to provide a better understanding of the creation of the universe and the forces that work in it. The advancements also provide an inexhaustible supply of electrical power using thermonuclear fusion reactions, reliable communication with space and reentry vehicles, propulsion devices appropriate for interplanetary travel, devices for producing and amplifying high-frequency radio energy, electrical generators with no mechanical parts and numerous semiconductor devices.

\section{Future Directions}


The immediate future must be devoted to a better understanding of the behavior of Plasmas and related phenomena under various conditions. The most significant progress is thus most likely to be made in fundamental studies under controlled conditions. It is difficult to predict what the long-term future will hold. However, we can predict that Plasma physics will grow both in importance and in scope. The present problems are indeed formidable, but the richness of the rewards for their solution warranted the interest and effort, which is and will be devoted to Plasma technology.

Plasma has played an important role in every field of life. The applications of Plasma are widespread in the fields of medicine, energy, environmental sciences, physics, etc. Plasma is present in human blood also and is the most abundant form of matter and these facts increase the importance of studies linked to Plasma.

We have discussed some of the applications of Plasma with respect to engineering and medical field. Other applications may include Plasma particle accelerator, Ion Propulsion of spacecraft, Plasma spray coating, corona dying of ink and textile, isotopes separation, ozone production for water purification, etc. As the developments in these applications occur, Plasma physics will become more important and will find many other practical uses and the role of engineering will become integral in the applications of Plasma.

\section{References}

1 J. DAWSON, and C. OBERMAN, "High-frequency conductivity and the emission and absorption coefficients of a fully ionized Plasma”, Phys. Fluids, 5(1962), 517.

2 J. DAWSON, and C. OBERMAN, "Fhys. Fluids", 6 (1963), 394.

3 SP1TZER, L. Jr., Physics of Fully Ionized Gases. Interscience Publishers, Inc., New York (1956), 76 and p. 83.

4 E. Stoffels, Y. Aranda, T. Whitmore, D. Seymour and J. Rees, “A Plasma needle generates nitric oxide," Plasma Sources Sci. Technol., 15 (2006), 501-506. DOI: 10.1088/ 0963-0252/15/3/028.

5 S. Kuo, "Portable Plasma sterilizer," U; 1.S. Patent Application 12/030982, (2008).

6 F. Chen, X. Huang, D.G Cheng, X. Zhan "Hydrogen production from alcohols and ethers via cold Plasma: A review” IJHE 39 (2014), 9036-9046

7 H. Conrads and M. Schmidt "Plasma generation and Plasma sources". PSST 4(2000), 44-54.

8 U. Kogelschatz “Atmospheric-pressure Plasma technology” PPCF 46 (2004), 63-75.

9 C. Tendero, C. Tixier, P. Tristant ,J. Desmaison, P. Leprince “Atmospheric-pressure Plasmas: a review" SpectrochimActa Part B Atomic Spectrosc 61(2006), 2-30.

10 M. Laroussi, T. Akan “Arc-free atmospheric pressure cold Plasma jets: a review” Plasma Process Polymer 9 (2007), 77-88.

11 A. Fridman, S. Nester, LA. Kennedy, A. Saveliev, O. Mutaf-Yardimci "Gliding arc gas discharge" Prog Energy Combust Sci 25(1998), 11-31.

12 Z. Bo, JH. Yan, XD. Li, Y. Chi, KF. Cen " Scale-up analysis and development of gliding arc discharge facility for volatile organic compounds decomposition” J Hazard Mater 155 (2008), 494501.

13 E. Tatarova, FM. Dias, E. Felizardo, J. Henriques, CM. Ferreira, Gordiets "Microwave Plasma torches driven by surface waves" PSST 17 (2008).

14 Mouawad, B., Soueidan, M., Fabregue, D., Buttay, C., Allard, B., Bley, V., ... \& Martin, C "Application of the spark plasma sintering technique to low-temperature copper bonding" IEEE Transactions on Components, Packaging and Manufacturing Technology, 2(2012), 553-560. 
15 E. Igenbergs. J. Sparer, M. Rott, Kh, G. Schmitt-Thomas "On the application of Plasma pulses generated by electromagnetic and electro thermal launchers for surface Treatment" Magnetics, IEEE Transactions 31(1995).

16 X. P. Wu, J.M. Shi, Z. S. Chen, and B. Xu, "A new Plasma antenna of beam forming" Progress In Electromagnetics Research 126 (2012), 539-553.

17 E. Hnatiuc, D. Astanei, M. Ursache, B. Hnautiuc "A Review over cold Plasma reactors and their applications" ICEPE (2012),497 - 502

18 J. Pelletier, A. Anders, "Plasma based ion implantation and deposition: A review of physics, technology, and applications" Plasma Science, IEEE Transactions 33(2005), 1944-1959.

19 F.F. Chen "Industrial Application of low temperature Plasma physics" AIP 6 (1995), 2164-2175

20 O.T. Loza, A.G. Shkvarunets, and P.S. Strelkov, "Experimental Plasma relativistic microwave electronics" IEEE transactions on Plasma science, 26 (1998).

21 Turchi, P.J. Los Alamos Nat, "Review of some Plasma gun techniques for fusion at Megagauss Energy Densities" IEEE transactions on Plasma science 38 (2010).

22 R.K.Y. Fu, P. K. Chu, X.B. Tian, S.Q. Yang "Ignition and dynamics of high-voltage glow discharge Plasma implantation" NIMPRB 242 (2006), 275-278

23 Alexey A. Goncharov, Ian G. Brown "Plasma devices based on Plasma lens - A review of result and applications" IEEE 4 (2007).

24 S. Kwak "A review of switch mode sustain drivers with resonant networks for Plasma display panels" IEEE transactions on Industrial Electronics 57(2010), 1624-1634.

25 U. Kogelschatz "Dielectric-barrier discharges: their history discharge physics, and industrial application" Plasma Chem Plasma Process 23 (2007).

26 BM. Penetrante "Non thermal Plasma techniques for pollution control "Springer Verlag (1994).

27 P. Talebizadeh, M. Babaie, R. Brown, H. Rahimzadeh, Z. Ristovski, M. Arai “The Role of nonthermalPlasma technique in NOX treatment: A review” RSER 40 (2014), 886-901

28 Alex.Karengin, Olga Shakhmatov, Alek. Karengin, N. Novikov "Plasma utilization of liquid radioactive waste" IEEE (2013), 1 - 4.

29 R. Rahman, H. Ibrahium, and Y. Hung, "Liquid radioactive wastes treatment: a review”, Water, 3 (2011), 551-565.

30 J. McLeanA, J. Becker, S. Boulyga, H. Dietze and A. Montaser, "Ultratrace and isotopic analysis of long-lived radionuclides by double-focusing sector field inductively coupled Plasma mass spectrometry using direct liquid sample introduction", International Journal of Mass Spectrometry,208(2011), 193-204.

31 L. Rubin, and J. Poate, "Ion implantation in silicon technology", Industrial Physicist, 9(2003), 12-15.

32 M. C. Roco, C. A. Mirkin, and M. C. Hersam, "Nanotechnology research directions for societal needs in2020: Summary of international study” J. Nano particle Res 13, (2011), 897-919.

33 K. Ostrikov "Plasma nanoscience: Basic concepts and applications of deterministic nano fabrication" Weinheim, Germany:Wiley-VCH (2008).

34 K. Ostrikov, U. Cvelbar, and A. B. Murphy, "Plasma nanoscience: setting directions, tackling grand challenges" J. Phys. D, Appl. Phys. 44(2011), 174.

35 M. Meyyappan, "Plasma nanotechnology: Past, present and future” J.Phys. D, Appl. Phys. 44 (2011), 174.

36 M. Shiratani, K. Koga, S. Iwashita, G. Uchida, N. Itagaki, and K. Kamataki "Nano factories in Plasma: Present status and outlook” J. Phys. D, Appl. Phys. 44 (2011). 
37 M. Stein, E. Lo, D. Castner and B.Ratner, "Plasma pencil atmospheric mass spectrometry detection of positive ions from micronutrients emitted from surfaces”, Analytical chemistry, 84(2012), 1572-1578.

38 J. Janča, L/ Zajíčková, M. Klíma and P. Slavíček, "Diagnostics and application of the high frequency Plasma pencil”, Plasma chemistry and Plasma processing, 21(2001), 565-579.

39 L. Bromberg, D. Cohn, A. Rabinovich and J. Heywood, "Emissions reductions using hydrogen from Plasmatron fuel converters", International Journal of Hydrogen Energy, 26(2001), 1115-1121.

40 D. Cohn, J. Bromberg, A. Rabinovich and N. Alexeev, "U.S. Patent No. 6,322,757. Washington, DC: U.S. Patent and Trademark Office", (2001).

41 Giovannucci, E., Ascherio, A., Rimm, E. B., Stampfer, M. J., Colditz, G. A., \& Willett, W. C. (1995). Intake of carotenoids and retino in relation to risk of prostate cancer. Journal of the national cancerinstitute, 87(23), 1767-1776.

42 A. Roddam, M. Duffy, F. Hamdy, A. Ward, J. Patnick and C. Price, NHS Prostate Cancer Risk Management Programme, "Use of prostate-specific antigen (PSA) isoforms for the detection of prostate cancer in men with a PSA level of 2-10 ng/ml: systematic review and meta-analysis", European urology, 48(2005), 386-399.

43 P. Gann, J. Ma, E. Giovannucci, W. Willett, F. Sacks, C. Hennekens and M. Stampfer,” Lower prostate cancer risk in men with elevated Plasma lycopene levels results of a prospective analysis", Cancer research, 59(1999), 1225-1230.

44 F. Samuel, "U.S. Patent No. 3,567,898”, Washington, DC: U.S. Patent and Trademark Office, (1971).

45 E. Couch Jr, N. Sanders, Z. Lu, L. Luo, L. and S. Stenfelt,” U.S. Patent No. 5,380,976. Washington, DC: U.S. Patent and Trademark Office", (1995).

46 U. Valbusa, C. Boragno and F. de Mongeot, "Nanostructuring surfaces by ion sputtering. Journal of Physics: Condensed Matter", 14 (2002), 8153.

47 Elsherbeni, A. Z. and A. A. Kishk "Modeling of cylindrical objects by circular dielectric and conducting cylinders" IEEE Trans. Antennas Propag. 40 (1992), 96- 99.

48 D. Flamm and O. Auciello, "Plasma deposition, treatment, and etching of polymers: the treatment and etching of polymers", R. d'Agostino (Ed.). Elsevier,(2012).

49 Dwyer, T. J., J. R. Greig, D. P. Murphy, J. M. Perina, and R. E. Pechacek "On the feasibility of using an atmospheric dischargePlasma as an RF antenna” IEEE Trans. Antennas Propag. 32(1984), $141-146$.

50 Brog, G. G., J. H. Harris, D. G. Miljak, and N. M. Martin, “Application of Plasma columns to radiofrequency antennas” Appl. Phys. Lett. 74 (1999), 3272-3274.

51 J.Mathew, R. A. Meger, R. F. Fernsler, D. P. Murphy, R.E. Pechacek, and W. M. Manheimer, "Electronically steerable Plasma mirror based radar antenna" 10th International Conference on Antenna and Propagation, 436 (1997), 1469-1473.

52 Cheng, Z. F., "Design and research on Plasma microwave reflector," Center for Space Science and Applied Research, (2010).

53 Elsherbeni, A. Z. and A. A. Kishk "Modeling of cylindrical objects by circular dielectric and conducting cylinders” IEEE Trans. Antennas Propag. 40 (1992), 96-99.

54 P. Fauchais and A. Vardelle "Thermal Plasmas” IEEE Trans. Plasma Sci. 25 (1997), 1258-1280.

55 M. Goldman and R. S. Sigmond “Corona and insulation” IEEE Trans. Elect. Insulation, 17 (1982), 90-105.

56 B. Eliasson and U. Kogelschatz, "Non-equilibrium volume Plasma chemical processing” IEEE Trans. Plasma Sci. 19 (1991), 1063-1077. 
57 J. Y. Jeong, S. E. Babayan, V. J. Tu, J. Park, R. F. Hicks, and G. S. Selwyn "Etching materials with an atmospheric-pressure Plasma jet” Plasma Source Sci. Technol. 7(1998), 282-285.

58 D. R. Wells and G. Schmidt "Observation of Plasma rotation produced by an electrodeless Plasma gun” Phys. Fluids, 6 (1963), 418-421.

59 P. J. Turchi, M. L. Alme, G. Bird, C. N. Boyer, S. K. Coffey, D. Conte, J. F. Davis, and S. W. Seiler "Review of Plasma flow switch development" IEEE Trans. Plasma S ci.15 (1987), 747-759.

60 I. G. Brown, Ed. “The Physics and Technology of Ion Sources”, (1989)

61 B. Wolf, "Handbook of Ion Sources". Boca Raton, FL: CRC, (1995).

62 Y. Carmel, W. R. Lou, T. M. Antonsen, J. Rodgers, B.Levush, W. W. Destler, and V. L. Granatstein "Studies of high power Plasma filled backward wave oscillators" Phys. Fluids B, 4 (1992), 22862292.

63 A. G. Shkvarunets, S. Kobayashi, J. Weaver, Y. Carmel, J. Rodgers, T. M. Antonsen, Jr., V. L. Granatstein, and W.W. Destler "Electromagnetic properties of corrugated and smooth waveguides filled with radially inhomogeneous Plasma".

64 E.B. Igenbergs, D.W. Jex, E.L. Shriver "New two stage accelerator for hypervelocity impact simulation", AIM Journal 13.

65 I. G. Brown, Ed.“The Physics and Technology of Ion Sources”. New York: Wiley (1989).

66 B. Wolf,"Handbook of Ion Sources”Boca Raton, FL: CRC, (1995)

67 S. Rosanvallon, C. Grisolia, G. Counsell, S. H. Hong,. Onofri, J. Worms,J. Winter, B. Annaratone, G. Maddaluno, and P. Gasior, "Dust controlintokamak environment," 8th Int. Symp. Fusion Nucl.Technol (2007), 1-5.

68 S. Benkadda and V. N. Tsytovich, "Excitation of dissipative drift turbulence in dustyPlasmas," Plasma Phys. Rep 28 (2002), 395-397.

69 S. I. Krasheninnikov and T. K. Soboleva, "Dynamics and transport of dust particles in tokamak edge Plasmas," Plasma Phys. Control. Fusion 47(2005), 339-352. 